\title{
Optical Third-Harmonic Generation in Graphene
}

\author{
Sung-Young Hong, ${ }^{1}$ Jerry I. Dadap, ${ }^{2, *}$ Nicholas Petrone, ${ }^{3}$ Po-Chun Yeh, ${ }^{4}$ James Hone, ${ }^{3}$ and Richard M. Osgood, Jr. ${ }^{2,4}$ \\ ${ }^{1}$ Department of Chemistry, Columbia University, New York, New York 10027, USA \\ ${ }^{2}$ Department of Applied Physics and Applied Mathematics, Columbia University, New York, New York 10027, USA \\ ${ }^{3}$ Department of Mechanical Engineering, Columbia University, New York, New York 10027, USA \\ ${ }^{4}$ Department of Electrical Engineering, Columbia University, New York, New York 10027, USA
}

(Received 8 April 2013; published 10 June 2013)

\begin{abstract}
We report strong third-harmonic generation in monolayer graphene grown by chemical vapor deposition and transferred to an amorphous silica (glass) substrate; the photon energy is in threephoton resonance with the exciton-shifted van Hove singularity at the $M$ point of graphene. The polarization selection rules are derived and experimentally verified. In addition, our polarization- and azimuthal-rotation-dependent third-harmonic-generation measurements reveal in-plane isotropy as well as anisotropy between the in-plane and out-of-plane nonlinear optical responses of graphene. Since the third-harmonic signal exceeds that from bulk glass by more than 2 orders of magnitude, the signal contrast permits background-free scanning of graphene and provides insight into the structural properties of graphene.
\end{abstract}

DOI: 10.1103/PhysRevX.3.021014

Subject Areas: Graphene, Optics

\section{INTRODUCTION}

Single-layer graphene has become a subject of intense interest and study because of its remarkable electronic, optical, mechanical, and thermal properties, combined with its unique electronic band structure [1-4]. Despite its monolayer-to-few-layer thickness, graphene offers an array of properties that are of interest for optical physics and devices. These properties include relatively flat optical absorption from around 0.5 to $1.5 \mathrm{eV}$, with a strong dopingdependent absorption edge and pronounced excitonic effects [5-9]; coupling of optical and mechanical properties in graphene membranes [10]; and plasmonic properties $[11,12]$. Such studies have underscored the importance of the linear optical properties of graphene [5-9,13-15]. In addition, measurements of optical carrier generation in graphene have led to the observation of strong hot-electron photoluminescence as well as new scattering phenomena involving highly excited carriers [16-18].

Recent theoretical investigations of nonlinear optical effects arising from interband electronic transitions have revealed that, despite graphene's single-atomic-layer thickness, its nonlinear optical response is particularly strong [19-21]. The potential of graphene as a functional nonlinear optical material has engendered many nonlinear optical studies. Second-order-nonlinear optical effects, particularly second-harmonic generation (SHG), have been investigated theoretically [22-25] and experimentally $[22,26]$. Because ideal freestanding monolayer graphene is

\footnotetext{
*jerry@cumsl.msl.columbia.edu
}

Published by the American Physical Society under the terms of the Creative Commons Attribution 3.0 License. Further distribution of this work must maintain attribution to the author(s) and the published article's title, journal citation, and DOI. centrosymmetric, its second-order nonlinear response vanishes within the dipole approximation [24]. In contrast, symmetry-allowed third-order nonlinear optical effects in graphene are remarkably strong, leading to studies that include saturable absorption [27-32], optical limiting [33,34], two-photon absorption [35], four-wave mixing (FWM) [36,37], and current-induced SHG [38]. In one notable FWM investigation, the authors have estimated the third-order nonlinear susceptibility of single-layer and multilayer graphene and demonstrated the capability of FWM for imaging of graphene using two input beams [36].

Third-harmonic generation (THG) is a third-order nonlinear process that provides three key advantages over FWM: (1) It can be carried out with a single-wavelength source, in contrast to the two-beam method of FWM; (2) for graphene, there is negligible hot-electronluminescence background (generated by the fundamental wave) at the much larger photon energy of the thirdharmonic $(\mathrm{TH})$ output wave relative to that of the input wave; and (3) for typical sources, THG has a potential for imaging with higher transverse resolution, because of its shorter output wavelength and cubic power dependence, than is possible with FWM or with the linear optical process of the same fundamental frequency. THG has also been demonstrated as a scanned-microscopy probe of interfaces with axial resolution of the order of the confocal parameter [39].

A recent study experimentally demonstrated THG from graphene for transitions occurring near the $K$ point and was carried out at normal-incidence angle; in that study, the authors report a quadratic dependence of THG on graphene layer number [40]. In contrast, the present work reports experimental THG from graphene under conditions in which the $\mathrm{TH}$ is in three-photon resonance with the $M$ 
point of graphene and at non-normal-incidence angle in order to provide access to other nonlinear susceptibility elements not accessible under a normal-incidence configuration, and to probe thicker films, in the case of multilayer graphene. In addition, we present a theoretical description of THG in graphene by considering the nonlinear slab geometry. We derive the polarization selection rules by taking into account the full symmetry of the tensor properties of graphene and the layer-number dependence of the $\mathrm{TH}$, and compare these properties with experiment. Our theoretical calculations predict subquadratic dependence on layer number in direct quantitative agreement with our experiment. Finally, we demonstrate the first imaging of discrete graphene crystals by THG.

Thus, our goal is twofold: to characterize the TH nonlinear optical response of graphene near its $M$ saddle point and to examine the potential of THG as an optical probe and imaging approach for graphene. Our study has yielded important physics insights in THG from graphene, including the following: (1) the isotropy of the in-plane nonlinear optical response, (2) anisotropy between the out-of-plane and in-plane nonlinear optical responses, and (3) the coherent nature of THG, which gives rise to an approximately subquadratic layer dependence of the THG signal at low layer numbers. In addition, the strong $\mathrm{TH}$ signals from graphene on amorphous silica glass $\left(\mathrm{SiO}_{2}\right)$ provide high contrast between graphene and glass, thereby permitting nearly background-free imaging of graphene islands, which uncovers thin-film structure that is difficult to observe via linear optical microscopy. Furthermore, the use of THG allows a broader choice of substrates than for typical optical imaging of graphene using certain fixed-thickness oxide layers on $\mathrm{Si}(001)$ to facilitate optical contrast [41]. This capability to probe graphene on arbitrary substrates is an important advantage of THG over linear optical imaging since graphene has been transferred to various substrates, including silicon-on-insulator [37], sapphire [42], diamondlike carbon [43], hexagonal boron nitride [44], quartz and glass [45], and flexible polymer substrates [46-49], for photonic and electronic applications.

\section{THEORETICAL CONSIDERATIONS}

We begin our description of the nonlinear optical response of graphene by considering the nonlinear optical process in a slab geometry for the two cases of the harmonic field perpendicular and parallel to the incidence plane ( $s$ - and $p$-polarized cases, respectively) as previously derived by Bloembergen and Pershan in their classic paper [50]. Specifically, we derive the linear and nonlinear optical fields arising from a nonlinear slab on a semi-infinite substrate. The extension of the model to the case with a finite substrate is straightforward but will be more complex, as has been considered in SHG from multilayers [51]. The simpler case of semi-infinite substrate, in which multiple reflections within the substrate are absent, is justified, since the coherence length of the fundamental beam, $\lambda^{2} /(n \Delta \lambda) \approx 21 \mu \mathrm{m}$, is much less than the substrate thickness, typically around $1 \mathrm{~mm}$ (as in our case). Figure 1 shows the optical geometry with the relevant fields and polarization. The nonlinear optical parameters are denoted by $F_{i}$, where $F=E$ (electric field), $H$ (magnetic intensity), $\mathbf{K}$ (nonlinear optical wave vector), or $\theta$ (wave-vector angle relative to surface-normal direction), and $i=$ $\left\{R, M, M^{\prime}, T\right\}$, indicating the reflected, internal downward-going, internal upward-going, and transmitted fields, respectively. In addition, the nonlinear polarization $P^{\mathrm{NL}}$, which is the complex amplitude of $\mathbf{P}^{\mathrm{NL}}$, is associated with the inhomogeneous wave vector $\mathbf{K}_{S}=3 \mathbf{k}$, where $\mathbf{k}$ is the downward-going linear wave vector in the slab; $\mathbf{K}_{S}$ is directed at the angle $\theta_{S}$ relative to the surface normal; and $\alpha$ is the angle between $\mathbf{P}^{\mathrm{NL}}$ and $\mathbf{K}_{S}$. The magnitude of the component parallel to the surface of each of the nonlinear wave vectors is equal to $K_{\|} \equiv K_{R, \|}$ (generalized Snell's law). The dielectric constants in the reflection, medium,

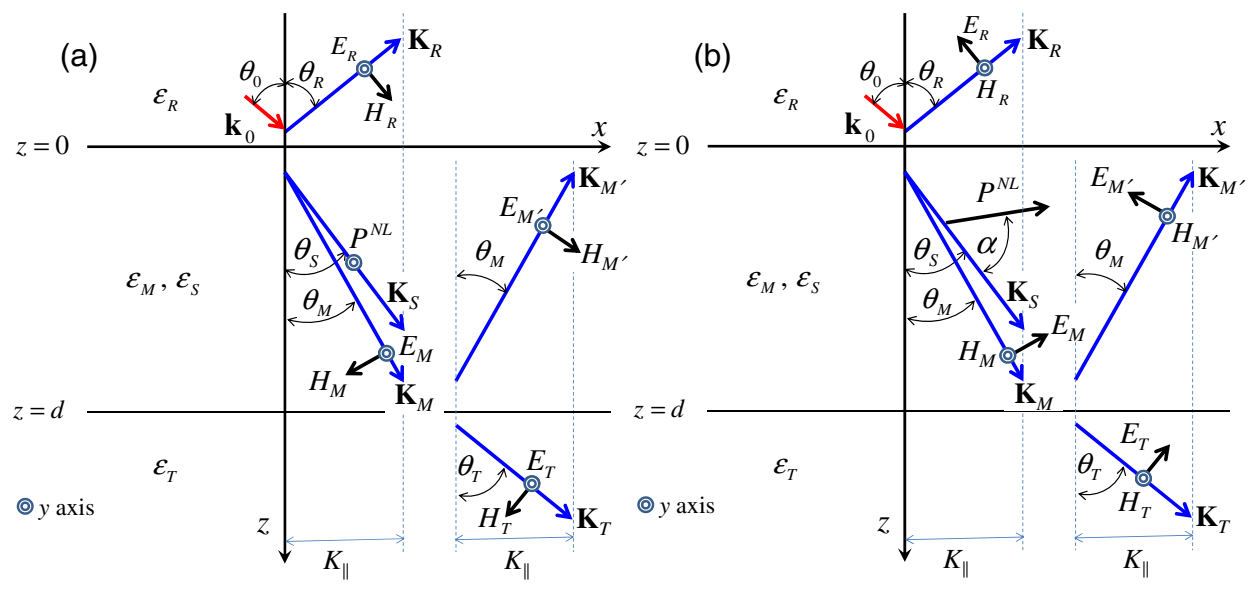

FIG. 1. Geometry of harmonic fields and nonlinear polarization arising from a slab of nonlinear material for (a) $s$ and (b) $p$ polarizations. The fundamental wave vector $\mathbf{k}_{0}$ is also shown for reference. 
and transmission regions are given by $\varepsilon_{R}, \varepsilon_{M}$, and $\varepsilon_{T}$, respectively, at the nonlinear optical frequency, $\Omega$, which we will set later to $3 \omega$ for the case of THG. Note that the dielectric constant associated with the nonlinear polarization, $\varepsilon_{S}=\varepsilon_{S}(\omega)$, is evaluated at the fundamental frequency.
For each of the perpendicular- and parallel-polarization configurations, a set of four linear equations is solved by applying the appropriate boundary conditions, as previously derived by Bloembergen and Pershan [50]. The relevant results are the expressions for the reflected harmonic field, $E_{R}$, for $s$ - and $p$-polarized cases, which are correspondingly given by [50]

$E_{R}^{\perp}=\frac{4 \pi P_{\perp}^{\mathrm{NL}}\left[A\left(n_{T} \cos \theta_{T}-n_{S} \cos \theta_{S}\right) n_{M} \cos \theta_{M}-B\left(n_{S} n_{T} \cos \theta_{S} \cos \theta_{T}-n_{M}^{2} \cos ^{2} \theta_{M}\right)\right]}{\left(n_{M}^{2}-n_{S}^{2}\right)\left[\exp \left(2 i \phi_{M}\right)\left(n_{R} \cos \theta_{R}-n_{M} \cos \theta_{M}\right)\left(n_{T} \cos \theta_{T}-n_{M} \cos \theta_{M}\right)-\left(n_{R} \cos \theta_{R}+n_{M} \cos \theta_{M}\right)\left(n_{T} \cos \theta_{T}+n_{M} \cos \theta_{M}\right)\right]}$

$E_{R}^{\|}=\frac{4 \pi P_{\|}^{\mathrm{NL}}\left\{\left[A\left(n_{S} \cos \theta_{T}-n_{T} \cos \theta_{S}\right) n_{M} \cos \theta_{M}-B\left(n_{M}^{2} \cos \theta_{T} \cos \theta_{S}-n_{T} n_{S} \cos ^{2} \theta_{M}\right)\right] n_{M} \sin \alpha-\left[A n_{T} \cos \theta_{M}+B n_{M} \cos \theta_{T}\right]\left(n_{M}^{2}-n_{S}^{2}\right) \sin \theta_{S} \cos \alpha\right\}}{n_{M}\left(n_{M}^{2}-n_{S}^{2}\right)\left[\exp \left(2 i \phi_{M}\right)\left(n_{M} \cos \theta_{R}-n_{R} \cos \theta_{M}\right)\left(n_{M} \cos \theta_{T}-n_{T} \cos \theta_{M}\right)-\left(n_{M} \cos \theta_{R}+n_{R} \cos \theta_{M}\right)\left(n_{M} \cos \theta_{T}+n_{T} \cos \theta_{M}\right)\right]}$

with phase terms

$$
\begin{aligned}
& A=1+\exp \left(2 i \phi_{M}\right)-2 \exp \left[i\left(\phi_{M}+\phi_{S}\right)\right], \\
& B=1-\exp \left(2 i \phi_{M}\right),
\end{aligned}
$$

where the relevant parameters are defined as $\phi_{M}=$ $n_{M} \Omega d / c, \phi_{S}=n_{S} \Omega d / c, n_{R}=\varepsilon_{R}^{1 / 2}(\Omega), n_{M}=\varepsilon_{M}^{1 / 2}(\Omega)$, $n_{T}=\varepsilon_{T}^{1 / 2}(\Omega)$, and $n_{S}=\varepsilon_{S}^{1 / 2}(\omega)$.

We now consider the complex amplitudes $P_{\perp}^{\mathrm{NL}}$ and $P_{\|}^{\mathrm{NL}}$ for the case of THG in Eqs. (1a) and (1b), which determine the components of the nonlinear optical polarization $\mathbf{P}^{\mathrm{NL}}$ according to Fig. 1 via the relations $P_{x}^{\mathrm{NL}}=P_{\|}^{\mathrm{NL}} \sin \left(\theta_{s}+\right.$ $\alpha), P_{y}^{\mathrm{NL}}=P_{\perp}^{\mathrm{NL}}$, and $P_{z}^{\mathrm{NL}}=P_{\|}^{\mathrm{NL}} \cos \left(\theta_{s}+\alpha\right)$. Monolayer graphene possesses $D_{6 h}(6 / \mathrm{mmm})$ symmetry; however, when supported on the surface of a glass substrate, graphene loses its inversion symmetry along the surface normal, thus giving rise to $C_{6 v}(6 \mathrm{~mm})$ symmetry. For either $D_{6 h}$ or $C_{6 v}$ symmetry, there are 21 nonzero $\chi^{(3)}$ elements, of which only ten are independent [52]; the Cartesian components $\quad P_{i}^{\mathrm{NL}}=\sum_{j} \sum_{k} \sum_{l} \chi_{i j k l}^{(3)} E_{j} E_{k} E_{l} \quad(\{i, j, k, l\}=$ $\{x, y, z\})$ of $\mathbf{P}^{\mathrm{NL}}$, with the graphene surface along the $x y$ plane, may be written as

$$
\begin{aligned}
P_{\{x, y\}}^{\mathrm{NL}} & =\chi_{1}(\mathbf{E} \cdot \mathbf{E}) E_{\{x, y\}}+\left(\chi_{1}^{\prime}-\chi_{1}\right) E_{\{x, y\}} E_{z}^{2}, \\
P_{z}^{\mathrm{NL}} & =\chi_{3}^{\prime}(\mathbf{E} \cdot \mathbf{E}) E_{z}+\left(\chi_{3}-\chi_{3}^{\prime}\right) E_{z}^{3},
\end{aligned}
$$

where four effective susceptibilities, $\chi_{1} \equiv \chi_{x x x x}^{(3)}=$ $\chi_{x x y y}^{(3)}+\chi_{x y x y}^{(3)}+\chi_{x y y x}^{(3)}, \chi_{1}^{\prime} \equiv \chi_{x x z z}^{(3)}+\chi_{x z x z}^{(3)}+\chi_{x z z x}^{(3)}, \chi_{3} \equiv$ $\chi_{z z z z}^{(3)}$, and $\chi_{3}^{\prime} \equiv \chi_{z z x x}^{(3)}+\chi_{z x z x}^{(3)}+\chi_{z x x z}^{(3)}$, are expressed in terms of the ten independent susceptibility elements (note that terms whose $x$ and $y$ indices are interchanged are equal, e.g., $\chi_{x x x x}^{(3)}=\chi_{y y y y}^{(3)}, \chi_{x x y y}^{(3)}=\chi_{y y x x}^{(3)}$, etc.); $E_{i}$ is the component of the downward-going electric-field amplitude $\mathbf{E}$ of the fundamental beam inside the medium. In principle, one needs to use both downward-going (E) and upward-going $\left(\mathbf{E}^{\prime}\right)$ fundamental waves in the slab. In the prescription of Bloembergen and Pershan, however, when $|E|>\left|E^{\prime}\right|$, the nonlinear polarization constructed solely on the downward-going field inside the slab (corresponding to
$E$ ) will approximate the correct result [50]; this case is satisfied in graphene because of its low linear reflectance.

As we will show experimentally below, the use of a circularly polarized input beam yields no TH signals and, consequently, second terms vanish in Eqs. (2a) and (2b) since $\mathbf{E} \cdot \mathbf{E}=0$, yielding $\chi_{1}=\chi_{1}^{\prime}$ and $\chi_{3}=\chi_{3}^{\prime}$. These conditions lead to the relations $\chi_{x x x x}^{(3)}=\chi_{x x y y}^{(3)}+\chi_{x y x y}^{(3)}+$ $\chi_{x y y x}^{(3)}=\chi_{x x z z}^{(3)}+\chi_{x z x z}^{(3)}+\chi_{x z z x}^{(3)}$ and $\chi_{z z z z}^{(3)}=\chi_{z z x x}^{(3)}+\chi_{z x z x}^{(3)}+$ $\chi_{z x x z}^{(3)}$, and reduce the number of independent tensor elements from 10 to 8 . Thus, the polarization can now be written simply as

$$
P_{i}^{\mathrm{NL}}=\chi_{i}(\mathbf{E} \cdot \mathbf{E}) E_{i}
$$

where $\chi_{i}=\left\{\chi_{1}, \chi_{1}, \chi_{3}\right\}$ for $i=\{x, y, z\}$. Equation (3) implies that there are now two effective TH susceptibilities as a consequence of vanishing circular-polarized-input $\mathrm{TH}$ signals. These two effective susceptibilities correspond to an in-plane isotropic response associated with $\chi_{1} \equiv \chi_{x x x x}^{(3)}$ and an out-of-plane response associated with $\chi_{3} \equiv \chi_{z z z z}^{(3)}$. Equation (3) also predicts that purely $p$ - or $s$-polarized pump input beams produce purely $p$ - or $s$-polarized TH beams, respectively; i.e., $p$-in/ $p$-out and $s$-in $/ s$-out signals are allowed, while $p$-in $/ s$-out and $s$-in/ $p$-out, together with the circular-in/ $(s$ or $p)$-out polarization configurations are forbidden.

The graphene band structure further simplifies the values of the nonlinear susceptibility elements. Since, in our case, the relevant conduction and valence bands of graphene are formed from $\pi$ orbitals of carbon atoms, the matrix elements associated with the resonant downward-3 $\omega$ transitions are dominated by an in-plane ( $x$ or $y$ ) polarization corresponding to the leading index of the susceptibility tensor element. The left inset in Fig. 2(a) shows the energy-band diagram and a schematic three-photon transition process for the conditions of our experiment. In this diagram, the exciton-shifted energy gap at the $M$ point is $4.6 \mathrm{eV}$ [7-9], which is approximately three-photon resonant with our fundamental photon energy of $1.57 \mathrm{eV}$. In addition, near the $M$ 
(a)

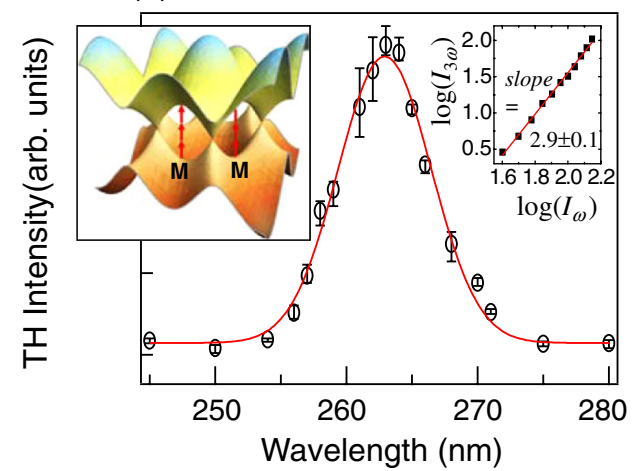

(b)

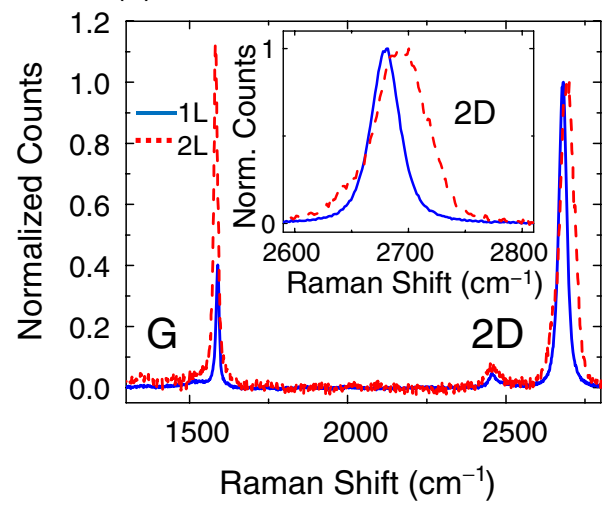

FIG. 2. (a) THG spectrum along with a right inset log-log plot showing a cubic power dependence of the TH signal intensity $I_{3 \omega}$ with respect to the intensity of the fundamental beam, $I_{\omega}$. The left inset shows the electronic band structure of graphene, including a three-photon resonance at the exciton-shifted graphene $M$ saddle point. (b) The Raman spectra in the monolayer (1L) and bilayer (2L) regions of the graphene flakes (solid and dashed curves, respectively). The Raman signals are normalized with respect to the $2 \mathrm{D}$ peak.

point, the usual linear graphene dispersion curve flattens, and thus the density of states is high because of the van Hove singularity at the saddle point, thereby enhancing the graphene nonlinear optical susceptibility via the transition matrix elements and the resonance corresponding to the denominator term. Transitions associated with the $z$ polarization are possible only with the $\sigma$ orbitals, in which the energy separation is much larger than those of the $\pi$ orbitals at the $M$ point by several electron volts [24]. Thus, for the three upward virtual $\omega$ transitions followed by the downward resonant $3 \omega$ transition in the vicinity of the $M$ point, $\left|\chi_{x x x x}^{(3)}\right| \gg\left|\chi_{z z z z}^{(3)}\right|$, and the remaining susceptibility terms that involve $z$ as the leading index are negligible. The number of independent elements is further reduced from 8 to 5 but only 1 effective tensor element, i.e., $\chi_{1}$, remains. Thus, we may set $\chi_{i} \approx$ $\left\{\chi_{1}, \chi_{1}, 0\right\}$ in Eq. (3). Since our transition occurs in the vicinity of the $M$ point, we anticipate that it would involve significant excitonic effects, as seen recently in single-photon transitions in this same region of $k$ space
[7-9]. As a result, it would be useful to examine this transition using full band-structure calculations of the nonlinear susceptibilities, in the presence of many-body effects.

\section{EXPERIMENT}

Single crystals of graphene were grown by chemical vapor deposition (CVD) and subsequently transferred onto glass substrates using procedures described in detail previously [53]. Briefly, graphene was grown at $1030{ }^{\circ} \mathrm{C}$ on $25-\mu \mathrm{m}$-thick copper foil, following low-pressure, encapsulated-growth methods [54], so as to yield spatially isolated single crystals with characteristic overall dimensions of approximately $200 \mu \mathrm{m}$. The graphene was subsequently transferred onto glass substrates (around $1 \mathrm{~mm}$ thick), which were first cleaned in a solution of sulfuric acid and hydrogen peroxide (3:1), utilizing a dry-transfer procedure with poly(methyl methacrylate) to support the graphene crystals throughout the transfer process; this procedure has been described previously in detail [53].

Our THG studies on the graphene samples used 50-fs, 789-nm pulses from a Ti:sapphire laser, which passed through a half-wave plate and polarizer and then focused on the sample with a typical average power of $100 \mathrm{~mW}$ at a $60^{\circ}$ incidence angle. The spot radii of the TH signal along its short and long dimensions are measured to be around 2.5 and $5 \mu \mathrm{m}$ using the knife-edge technique with a gold film, corresponding to a typical fundamental beam fluence of approximately $1 \mathrm{~mJ} / \mathrm{cm}^{2}$. At this fluence, no degradation of the graphene during irradiation was observed, thus permitting multiple and reproducible scans of the graphene flakes with no discernible change in signal levels. For the TH measurements, the sample is mounted on a mated orthogonal-translation and rotation stage, which permits 2D scanning as well as measurements of the rotational anisotropy of the TH signals.

The reflected THG signal was collected by a collimating lens and passed through an analyzer and then a PellinBroca prism to filter out the fundamental beam and a monochromator, before being detected by a photomultiplier tube. A monochromator, whose throughput was approximately $10 \%$ because of its small-bandwidth window centered at the peak THG signal, was necessary to reduce background signal significantly to the level of the dark counts. The conversion efficiency of the graphene-glass system is of the order of $10^{-13}$ relative to the incident power of the fundamental beam, and hence, we used a photon counting system for signal detection. The $p$-in/ $p$-out photon count rates for the glass substrate after background subtraction range from 0.2 to $0.8 \pm 0.5 / \mathrm{s}$, which are smaller than the background (noise level) counts of approximately $1.5 \pm 0.3 / \mathrm{s}$. After filtering, the TH rawsignal counts from the graphene-glass system were of the order of 100 counts/s per point. This count rate implies that to scan an image from a $1-\mathrm{mm}^{2}$ area, it will take $>10$ hours 
to scan with $5-\mu \mathrm{m}$ step size. If the fluence is kept fixed, improvements in signal counts at smaller spot sizes are possible through the use of shorter pulse widths, normalincidence geometry, and increased repetition rates; together with high-throughput spectral filters and spectral integration, we estimate that count rates $>10^{4} / \mathrm{s}$ are possible. Thus, faster scanning times, $<1$ hour, are achievable, which can be reduced further using 2D-imaging systems. As a point of comparison, we also carried out SHG measurements under the same laser and focusing parameters, which yielded signals that are 2 orders of magnitude weaker than those of THG.

Prior to conducting our THG measurements, Raman spectroscopy, with a 532-nm pump laser, was employed to establish the monolayer quality of our graphene. For purposes of comparison with previous Raman spectroscopic analyses of graphene, CVD graphene samples were also transferred onto $\mathrm{Si}(001)$ substrates with 300 nm-thick thermally grown $\mathrm{SiO}_{2}$, utilizing identical processing procedures as described above for glass substrates. Figure 2(b) shows Raman spectra for graphene crystals. For most of the graphene-crystal area except for a $20-\mu \mathrm{m}$ region approximately in its center, the absence of a $D$ peak and a $2 \mathrm{D} / \mathrm{G}$ ratio greater than 2 indicated a monolayer crystal of high quality. Within the central spot of a graphene flake, the $2 \mathrm{D} / \mathrm{G}$ ratio is less than 1 and the $2 \mathrm{D}$ peak is broader compared to the monolayer case (inset), in agreement with previous Raman measurements of bilayer graphene films $[55,56]$. Thus, this central region contains a bilayer film, a result observed also for CVD-grown graphene on $\mathrm{Cu}$ foils [57]. Raman spectra were also taken on graphene flakes transferred to the silica substrate used in our THG studies, with identical results.

\section{RESULTS AND DISCUSSION}

For our THG studies, when the fundamental beam irradiated the graphene crystal, strong nonlinear optical emission was observed. To verify the origin of the signal, we detected a TH signal at multiple locations over different graphene crystals. Typical TH intensity-dependence measurements (right inset) at one location are shown in Fig. 2(a). The wavelength of the TH spectrum centered at $263 \pm 4 \mathrm{~nm}$ and its $I_{\omega}^{3}$ power dependence fully confirm the nature of the nonlinearity of the signal. The width of the $\mathrm{TH}$ frequency spectrum is close to $3^{1 / 2}$ of that of the fundamental beam, assuming a Gaussian linewidth and taking into account instrumental broadening. Note the absence of any photoluminescence background.

To elucidate the symmetry properties of graphene with regards to its nonlinear optical response, we compared the relative magnitudes of the signals from graphene-glass to that of a glass surface using different polarization combinations, as shown in Fig. 3. For the case of the grapheneglass surface, the dominant signals arise from the $s$-in/s-out and $p$-in/ $p$-out polarization combinations. An

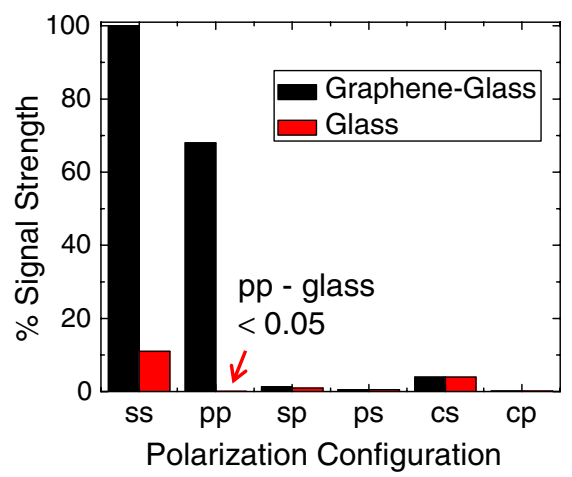

FIG. 3. Relative signal levels at various THG polarization combinations normalized to the $s$-in/s-out signal levels of the graphene-glass system, which confirm the third-order nonlinear optical response of graphene.

analysis of the signal levels for these two configurations implies anisotropy between the in-plane and out-of-plane nonlinear optical responses of graphene. The crosspolarized configurations as well as the circularly polarized input $(c$-in $/ s$-out, $c$-in $/ p$-out $)$ data are seen to be weak relative to the dominant signals. It should be noted that, in general, a circularly polarized beam may change in ellipticity as the beam enters the material. Inside the graphene layer, one needs to take into account the Fresnel transmission coefficients for each orthogonal component of the field. Using the refractive indices of graphene and the oxide substrate, we find that the ratio of the magnitudes and the relative phase of the $s$ and $p$ components of the fundamental field inside the graphene layer are nearly unchanged; i.e., the field maintains its circularly polarized character. We calculate the ratio of the circularly polarized intensity signal to that of the linearly polarized case to be of the order of $10^{-6}$.

These results clearly indicate that the expected THG polarization selection rules for the $C_{6 v}$ symmetry, as discussed above, are present. In addition, a comparison between the glass and graphene-glass signals for the $p$-in/ $p$-out configuration indicates a negligible contribution from the glass substrate. This difference in signal levels between glass and graphene exceeds 2 orders of magnitude, and hence the total signal appears as arising entirely from the graphene layer for this polarization configuration. In addition, for imaging purposes, we have used the $p$-in/ $p$-out configuration to maximize the contrast between the graphene and glass signals.

Additional properties of the TH generation could be established via probing of an array of similar graphene films. Figure 4(a) shows a scanned image, obtained from a large area $\left(1.4 \times 1.4 \mathrm{~mm}^{2}\right)$ on a sample using the $p$-in/ $p$-out configuration. The image contains randomly distributed star-shaped graphene crystals, with nearly uniform overall dimensions of approximately $200 \mu \mathrm{m}$. In this constellation of graphene "stars," there are clear "bright spots" in the center of most stars, which are attributed to 

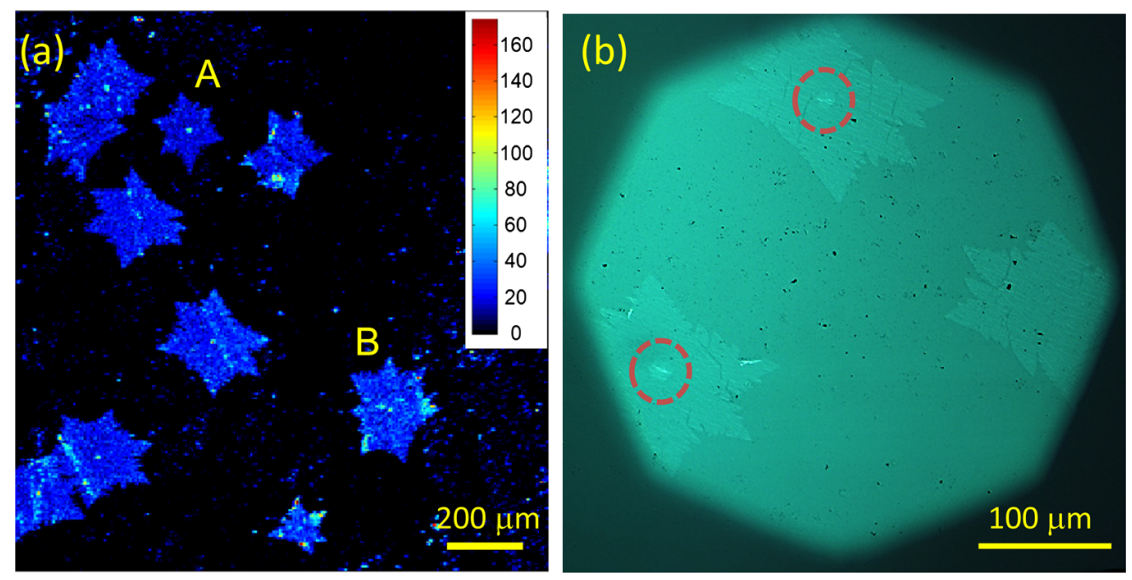

FIG. 4. (a) THG $p$-in/ $p$-out scan of a $1.4 \times 1.4 \mathrm{~mm}^{2}$ area featuring star-shaped CVD-grown monolayer graphene crystals on glass, which are approximately $200 \mu \mathrm{m}$ in characteristic dimension. (b) Optical microscopy of graphene stars on glass. The central bright spots (inside the dashed red circles) correspond to bilayer graphene.

bilayer signals from our Raman data and are approximately $20 \mu \mathrm{m}$ in diameter; see also a magnified view of one of the stars in Fig. 4(a). These results in the central region are consistent with both optical imaging [Fig. 4(b)], in which the bilayer regions are clearly visible, and Raman measurements [Fig. 2(b)]. Note also that the high-contrast TH signals from the graphene stars are further enhanced because of the absence of $p$-in/ $p$-out TH signals from the glass substrate at this incidence angle.

A higher-resolution scan of a single graphene flake, denoted by "A" in Fig. 4(a), is presented in Fig. 5(a) for a $240 \times 240 \mu \mathrm{m}^{2}$ scanning field. The bright spot at its center enables us to quantify the dependence of the relative $\mathrm{TH}$ signal level as a function of layer number relative to the rest of the graphene signals. In particular, two line scans are shown in Figs. 5(b) and 5(c), corresponding to the vertical and horizontal dashed lines in Fig. 5(d), which pass through the central spot of this graphene flake. The peak TH signal, which is located at the central spot of this graphene star, is larger by a factor of approximately $3.8 \pm 0.1$ than the average signal from the remainder of the graphene crystal. As discussed above, our Raman measurements of this star on this substrate indicate that the central region has a bilayer composition. Using the reflected field from Eq. (1b) to calculate the intensity $I \propto\left|E_{R}^{\|}\right|^{2}$ as a function of graphene layer number $N$, and making use of the optical constants of graphene at the fundamental and TH wavelengths [15], $n_{\mathrm{Gr}}(\lambda) \approx$ $3+1.5 i$ and $n_{\mathrm{Gr}}(\lambda / 3) \approx 2+3 i$, respectively, and a slab thickness $d=3.35 \mathrm{~N} \AA$, we calculate a signal ratio of 3.7 between the bilayer and monolayer regions, in close agreement with our results. As a check, these constants are used
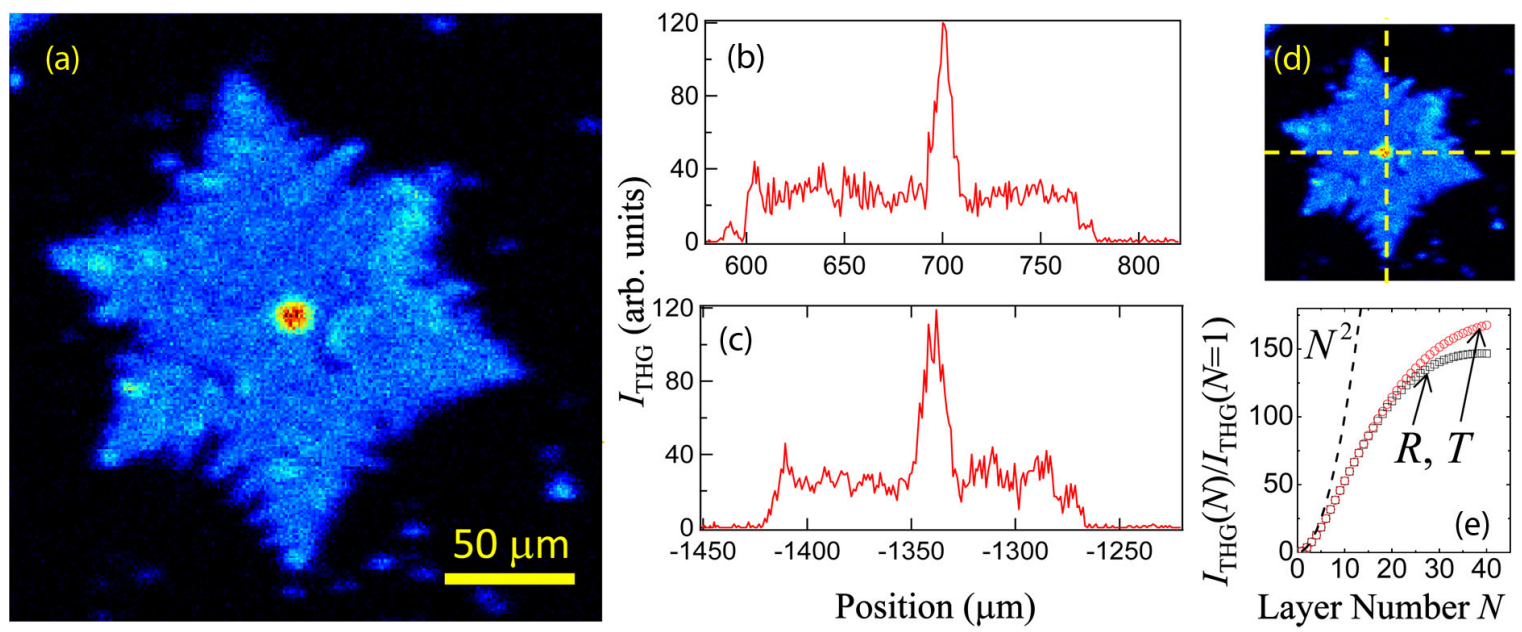

FIG. 5. (a) TH $p$-in/p-out scan of graphene star "A" shown in Fig. 4(a). (b) and (c) Line-scan data along the dashed vertical and horizontal lines that pass through the central spot, as shown in (d). (e) calculated layer dependence of THG for reflected signals $(R)$ using Eq. (1b) and transmitted ( $T$ ) signals normalized to a monolayer signal; quadratic dependence is shown for reference (dashed). 
to calculate normal-incidence reflectance and transmittance values using the linear optical analogue of either Eq. (1a) or Eq. (1b) for the case of an air-graphene-air system with $n_{R}=n_{T}=1$ at our fundamental wavelength. This calculation yields $R \approx 0$ and $T \approx 2.4 N \%(N<6)$, respectively, as expected for multilayers of graphene and in good agreement with the measured values for suspended graphene in Ref. [5].

For comparison, the normal-reflection FWM signal for visible to near-IR pump wavelengths is related to the layer number according to the relation given by $I_{\mathrm{FWM}} \propto$ $N^{2} /\left(1+N \pi \alpha_{F} / 2\right)^{8}$ [36], where $\alpha_{F}$ is the fine-structure constant and $\pi \alpha_{F} \approx 0.023$ is the single-layer absorbance of graphene; this formula yields a ratio of $I_{\mathrm{FWM}}(N=2)$ / $I_{\text {FWM }}(N=1)=3.7$, which is similar to our measurements. Note that in the absence of absorption for the fundamental and output wavelengths, the ratio between $N$-layer and monolayer signals should be $N^{2}$ for either the THG or FWM process, and hence for the case of bilayer signals, this ratio equals 4 . For larger $N$ values, the effect of absorption on both input and output waves becomes stronger, and consequently, the THG intensity initially increases subquadratically with layer number up to a certain layer number where the signal reaches a maximum value and then decreases at larger layer numbers. The layer number corresponding to the maximum signal depends on the incidence angle. Assuming that the dielectric constants are independent of layer number, we estimate that as high as about 40 layers can be determined with THG at the current incidence angle [see Fig. 5(e)]. This number is reduced to around 20 layers under normal incidence, similar to that estimated via FWM [36]. This general behavior is also true for the transmitted TH signal. With Raman imaging, however, this limit is around 10 where the signal is indistinguishable from graphite [58].

The high contrast in the TH signal, described above for Fig. 4, has important consequences for higher-resolution imaging. As a specific example, high contrast makes it particularly easy to observe certain structural features in Fig. 5(a), such as the fractal-like topographical structures, wrinkling, and depressions at the edge and internal area of the graphene star. These structures observed using THG follow similar features that are obtained using atomic force microscopy (AFM) on transferred CVD-grown graphene [59]. We are in the process of carrying out studies to correlate features observed using THG with that observed through AFM. Such structural features are also seen using optical microscopy but without the high contrast, as shown in Fig. 4(b); this result is due to the significantly lower contrast of linear optical microscopy. In addition, the image also shows internal structure, which is not readily apparent in linear microscopy. In particular, fluctuations in the image brightness are seen in Fig. 5(a). Thus, we speculate that topographic variations in the graphene may cause changes in the local optical electric field, which is then magnified by the nonlinear interactions, although it is not possible to rule out the interplay of other effects such local fluctuations in doping, which varies the electronic response, or local strain. Note, in addition, that despite the use of a modest numerical aperture (NA) in our laser-beam imaging system, such spatial features are observed because of the inherent improved spatial resolution arising from the $I_{\omega}^{3}$ dependence of the TH signal, as well as the shorter TH wavelength. Further significant improvement in spatial resolution can be expected by making use of a higher NA system to enable submicrometer resolution. Another important consequence of our result is the potential use of TH imaging of graphene for arbitrary substrates beyond the now-standard 300-nm oxide-silicon substrate [41]. Such alternative substrates are currently a topic of interest brought about by new graphene-substrate combinations made possible with CVD graphene, as discussed above.

As a final test of the in-plane isotropic nonlinear response of graphene to THG, we performed azimuthally dependent experiments by rotating the sample in the plane of the graphene. Figure 6 shows rotational scans of the graphene flake denoted as B in Fig. 4(a); each image is taken at a different azimuthal angle, spaced by increments of $10^{\circ}$. We chose this particular graphene flake because of the presence of bright areas of nonuniform TH signals, away from the center of the flake. These spots provide guide points to help track the rotation of the graphene flake. Note that the magnitude of the signals at an arbitrary local point within the star is generally constant to within $5 \%$ at all the angles used, indicating the isotropic response of the third-order nonlinearity of graphene. The results of these rotational measurements are consistent with the fact that signals among all the stars are relatively uniform in Fig. 4(a), despite the random angular crystallographic orientation of the stars. Finally, we note that this series of scans also demonstrates the high reproducibility of the THG microscopy of graphene.

In order to better quantify the relative strength of the TH signal from graphene, we also measured the corresponding $\mathrm{TH}$ signals from gold and other $\mathrm{SiO}_{2}$-based substrates. Gold has also been utilized as a reference substrate in a previous FWM study of graphene [36]. For our gold samples, we used a high-purity $100-\mu \mathrm{m}$-thick gold film evaporated on a glass substrate. From our measurements and from calculations taking into account the fields within the respective media, we obtained $\left|\chi_{1 \text {, graphene }} / \chi_{1 \text {, gold }}\right| \sim 4$.6; we can further deduce the ratio of their nonlinearity per unit thickness as $R \equiv$ $\left(\left|\chi_{1 \text {,graphene }}\right| / L_{\text {graphene }}\right) /\left(\left|\chi_{1 \text {,gold }}\right| / L_{\text {gold }}\right) \sim 1.6 \times 10^{2}$, where the corresponding graphene and gold thicknesses were taken as $L_{\text {graphene }}=0.335 \mathrm{~nm}$ and $L_{\text {gold }}=12 \mathrm{~nm}$, respectively. Here, we have taken the gold thickness as the inverse of its tabulated absorption coefficient at the TH wavelength [60]. These results show that the nonlinearity per unit thickness of graphene is particularly 

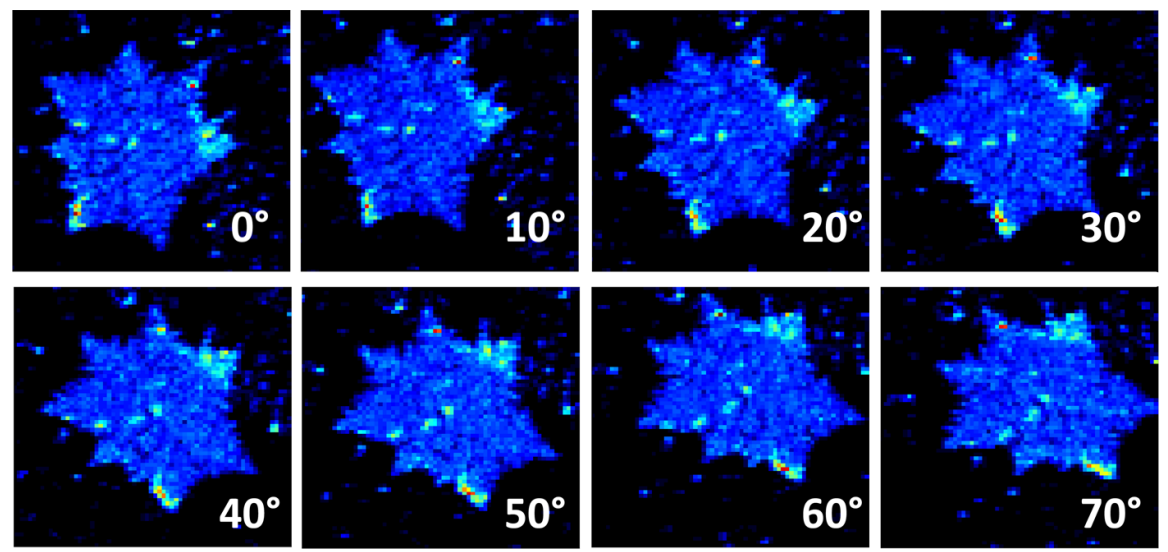

FIG. 6. Azimuthal dependence of THG scans of the graphene star "B" shown in Fig. 4(a), indicating the isotropy of the in-plane nonlinear optical response of graphene.

strong in comparison with that of gold. Previously, the ratio $R$ was measured using FWM in the visible spectral range and away from the $M$ point of graphene [36]. As a comparison, our value of $R$, which is measured at the three-photon resonant $M$ point using the THG process, is about 4 times larger than the previous FWM measurement, i.e., $R_{\mathrm{THG}} / R_{\mathrm{FWM}} \approx 4$.

For the case of $\mathrm{SiO}_{2}$-based substrates such as crystalline quartz, fused silica, and soda-lime glass, we found that the nonlinear susceptibility $\left|\chi_{1}\right|=\left|\chi_{x x x x}^{(3)}\right|$ values of these materials are within $20 \%$ of each other. In extracting these parameters, we made use of the corresponding expression to Eq. (1a) for the case of two media [50], given by $E_{R}^{\perp}=-4 \pi P_{\perp}^{\mathrm{NL}}\left(n_{M} \cos \theta_{M}-n_{S} \cos \theta_{S}\right) /\left[\left(n_{M}^{2}-n_{S}^{2}\right) \times\right.$ $\left.\left(n_{R} \cos \theta_{R}+n_{M} \cos \theta_{M}\right)\right]$. Our results show that the ratio $\left|\chi_{1, \text { graphene }} / \chi_{1, \mathrm{SiO}_{2}}\right|$ ranges from around 0.8 to $0.9 \times 10^{3}$; i.e., the graphene nonlinearity is nearly 3 orders of magnitude larger than that of the $\mathrm{SiO}_{2}$-based materials, which includes crystalline quartz.

\section{CONCLUSION}

In conclusion, we have demonstrated optical thirdharmonic generation as a deterministic probe of graphene's nonlinear optical properties. We have shown that, despite its typical use as a probe of bulk media, THG can also be a probe of the single-atom-thick graphene film with high discrimination due to the strong nonlinearity of graphene. The use of THG confirms the symmetry properties of graphene: Polarization-dependent and azimuthal-rotation measurements reveal in-plane isotropic symmetry as well as the anisotropy between the in-plane and out-of-plane nonlinear optical responses, in agreement with the hexagonal symmetry of graphene. These polarization-dependent measurements establish the relationships between tensor elements, which further allow us to reduce the number of independent tensor components. Together with the knowledge of band structure, the number of independent components reduces from 10 to 5 , and the number of effective measurable tensor component reduces from 4 to 1 , given by $\chi_{x x x x}^{(3)}$. The coherent nature of THG gives rise to a subquadratic layer dependence of the THG signal at low layer numbers. In addition, we have found that the magnitude of the nonlinear susceptibility $\chi_{x x x x}^{(3)}$ of graphene is nearly 5 times that of gold, and nearly 3 orders of magnitude larger than that of $\mathrm{SiO}_{2}$-based substrates including quartz crystal. This result shows the strong nonlinear optical response of the TH process for graphene, making it a potential probe for electronic processes near the excitonshifted $M$ point of graphene.

The in-plane isotropy, in conjunction with the coherent nature of THG, permits probing of the physical structure of graphene without crystallographic orientation dependence. THG further provides the potential for characterization of CVD-grown graphene structures on arbitrary substrates, without having to utilize high visibility contrast microscopy (i.e., not limited to $\mathrm{SiO}_{2} / \mathrm{Si}$ substrates) and without the graphene hot-electron luminescence background, as seen in other nonlinear optical studies such as FWM. Clearly, we have demonstrated that THG can be used as an optical microscopy probe of graphene even with modest optical focusing. In fact, THG has intrinsically high spatial resolution due to the cubic intensity dependence of the nonlinear process and its short TH wavelength.

\section{ACKNOWLEDGMENTS}

We thank Tony Heinz and Arend van der Zande for useful discussions. This work was funded by the AFOSR MURI Program on new graphene materials technology, Grant No. FA9550-09-1-0705. Nonlinear-optical laser experiments by S. Y.H. were supported as part of the Center for Redefining Photovoltaic Efficiency through MolecularScale Control, an Energy Frontier Research Center funded by the U.S. Department of Energy (DOE), Office of Science, Office of Basic Energy Sciences under Grant No. DE-SC0001085. 
[1] V. Singh, D. Joung, L. Zhai, S. Das, S. I. Khondaker, and S. Seal, Graphene Based Materials: Past, Present and Future, Prog. Mater. Sci. 56, 1178 (2011).

[2] M. J. Allen, V.C. Tung, and R. B. Kaner, Honeycomb Carbon: A Review of Graphene, Chem. Rev. 110, 132 (2010).

[3] S. D. Sarma, S. Adam, E. Hwang, and E. Rossi, Electronic Transport in Two-Dimensional Graphene, Rev. Mod. Phys. 83, 407 (2011).

[4] M. S. Fuhrer, C. N. Lau, and A. H. MacDonald, Graphene: Materially Better Carbon, MRS Bull. 35, 289 (2010).

[5] R. Nair, P. Blake, A. Grigorenko, K. Novoselov, T. Booth, T. Stauber, N. Peres, and A. Geim, Fine Structure Constant Defines Visual Transparency of Graphene, Science 320, 1308 (2008).

[6] Z. Li, E. Henriksen, Z. Jiang, Z. Hao, M. Martin, P. Kim, H. Stormer, and D. N. Basov, Dirac Charge Dynamics in Graphene by Infrared Spectroscopy, Nat. Phys. 4, 532 (2008).

[7] K.F. Mak, J. Shan, and T.F. Heinz, Seeing Many-Body Effects in Single- and Few-Layer Graphene: Observation of Two-Dimensional Saddle-Point Excitons, Phys. Rev. Lett. 106, 046401 (2011).

[8] V. Kravets, A. Grigorenko, R. Nair, P. Blake, S. Anissimova, K. Novoselov, and A. Geim, Spectroscopic Ellipsometry of Graphene and an Exciton-Shifted van Hove Peak in Absorption, Phys. Rev. B 81, 155413 (2010).

[9] L. Yang, J. Deslippe, C.-H. Park, M. L. Cohen, and S. G. Louie, Excitonic Effects on the Optical Response of Graphene and Bilayer Graphene, Phys. Rev. Lett. 103, 186802 (2009).

[10] R. A. Barton, I. Storch, V. P. Adiga, R. Sakakibara, B. R. Cipriany, R. Ilic, S. P. Wang, P. Ong, P. L. McEuen, and J. M. Parpia, Photothermal Self-Oscillation and Laser Cooling of Graphene Optomechanical Systems, Nano Lett. 12, 4681 (2012).

[11] L. Ju, B. Geng, J. Horng, C. Girit, M. Martin, Z. Hao, H. A. Bechtel, X. Liang, A. Zettl, and Y.R. Shen, Graphene Plasmonics for Tunable Terahertz Metamaterials, Nat. Nanotechnol. 6, 630 (2011).

[12] H. Yan, X. Li, B. Chandra, G. Tulevski, Y. Wu, M. Freitag, W. Zhu, P. Avouris, and F. Xia, Tunable Infrared Plasmonic Devices Using Graphene/Insulator Stacks, Nat. Nanotechnol. 7, 330 (2012).

[13] M. Bruna and S. Borini, Optical Constants of Graphene Layers in the Visible Range, Appl. Phys. Lett. 94, 031901 (2009).

[14] J. Weber, V. Calado, and M. Van de Sanden, Optical Constants of Graphene Measured by Spectroscopic Ellipsometry, Appl. Phys. Lett. 97, 091904 (2010).

[15] A. Matković, U. Ralević, G. Isić, M. Jakovljević, B. Vasić, I. Milošević, D. Marković, and R. Gajić, Spectroscopic Ellipsometry and the Fano Resonance Modeling of Graphene Optical Parameters, Phys. Scr. T149, 014069 (2012).

[16] C. H. Lui, K. F. Mak, J. Shan, and T. F. Heinz, Ultrafast Photoluminescence from Graphene, Phys. Rev. Lett. 105, 127404 (2010).

[17] R. J. Stöhr, R. Kolesov, J. Pflaum, and J. Wrachtrup, Fluorescence of Laser-Created Electron-Hole Plasma in Graphene, Phys. Rev. B 82, 121408 (2010).
[18] W.-T. Liu, S. Wu, P. Schuck, M. Salmeron, Y. Shen, and F. Wang, Nonlinear Broadband Photoluminescence of Graphene Induced by Femtosecond Laser Irradiation, Phys. Rev. B 82, 081408 (2010).

[19] K. L. Ishikawa, Nonlinear Optical Response of Graphene in Time Domain, Phys. Rev. B 82, 201402 (2010).

[20] S. Jafari, Nonlinear Optical Response in Gapped Graphene, J. Phys. Condens. Matter 24, 205802 (2012).

[21] H. Avetissian, A. Avetissian, G. Mkrtchian, and K. V. Sedrakian, Creation of Particle-Hole Superposition States in Graphene at Multiphoton Resonant Excitation by Laser Radiation, Phys. Rev. B 85, 115443 (2012).

[22] J. J. Dean and H. M. van Driel, Graphene and Few-Layer Graphite Probed by Second-Harmonic Generation: Theory and Experiment, Phys. Rev. B 82, 125411 (2010).

[23] S. Mikhailov, Theory of the Giant Plasmon-Enhanced Second-Harmonic Generation in Graphene and Semiconductor Two-Dimensional Electron Systems, Phys. Rev. B 84, 045432 (2011).

[24] M. Glazov, Second Harmonic Generation in Graphene, JETP Lett. 93, 366 (2011).

[25] S. Wu, L. Mao, A. M. Jones, W. Yao, C. Zhang, and X. Xu, Quantum-Enhanced Tunable Second-Order Optical Nonlinearity in Bilayer Graphene, Nano Lett. 12, 2032 (2012).

[26] J. J. Dean and H. M. van Driel, Second Harmonic Generation from Graphene and Graphitic Films, Appl. Phys. Lett. 95, 261910 (2009).

[27] Q. Bao, H. Zhang, Y. Wang, Z. Ni, Y. Yan, Z.X. Shen, K. P. Loh, and D. Y. Tang, Atomic-Layer Graphene as a Saturable Absorber for Ultrafast Pulsed Lasers, Adv. Funct. Mater. 19, 3077 (2009).

[28] W. B. Cho, J. W. Kim, H. W. Lee, S. Bae, B. H. Hong, S. Y. Choi, I. H. Baek, K. Kim, D.-I. Yeom, and F. Rotermund, High-Quality, Large-Area Monolayer Graphene for Efficient Bulk Laser Mode-Locking Near $1.25 \mu \mathrm{m}$, Opt. Lett. 36, 4089 (2011).

[29] D. Popa, Z. Sun, F. Torrisi, T. Hasan, F. Wang, and A. Ferrari, Sub 200 fs Pulse Generation from a Graphene Mode-Locked Fiber Laser, Appl. Phys. Lett. 97, 203106 (2010).

[30] Y.-W. Song, S.-Y. Jang, W.-S. Han, and M.-K. Bae, Graphene Mode-Lockers for Fiber Lasers Functioned with Evanescent Field Interaction, Appl. Phys. Lett. 96, 051122 (2010).

[31] W. Tan, C. Su, R. Knize, G. Xie, L. Li, and D. Tang, Mode Locking of Ceramic Nd: Yttrium Aluminum Garnet with Graphene as a Saturable Absorber, Appl. Phys. Lett. 96, 031106 (2010).

[32] J.-L. Xu, X.-L. Li, Y.-Z. Wu, X.-P. Hao, J.-L. He, and K.-J. Yang, Graphene Saturable Absorber Mirror for UltraFast-Pulse Solid-State Laser, Opt. Lett. 36, 1948 (2011).

[33] J. Wang, Y. Hernandez, M. Lotya, J. N. Coleman, and W. J. Blau, Broadband Nonlinear Optical Response of Graphene Dispersions, Adv. Mater. 21, 2430 (2009).

[34] M. Feng, H. Zhan, and Y. Chen, Nonlinear Optical and Optical Limiting Properties of Graphene Families, Appl. Phys. Lett. 96, 033107 (2010).

[35] H. Yang, X. Feng, Q. Wang, H. Huang, W. Chen, A. T. Wee, and W. Ji, Giant Two-Photon Absorption in Bilayer Graphene, Nano Lett. 11, 2622 (2011). 
[36] E. Hendry, P. Hale, J. Moger, A. Savchenko, and S. Mikhailov, Coherent Nonlinear Optical Response of Graphene, Phys. Rev. Lett. 105, 097401 (2010).

[37] T. Gu, N. Petrone, J. McMillan, A. van der Zande, M. Yu, G.-Q. Lo, D.-L. Kwong, J. Hone, and C.W. Wong, Regenerative Oscillation and Four-Wave Mixing in Graphene Optoelectronics, Nat. Photonics 6, 554 (2012).

[38] A. Y. Bykov, T. V. Murzina, M. G. Rybin, and E.D. Obraztsova, Second Harmonic Generation in Multilayer Graphene Induced by Direct Electric Current, Phys. Rev. B 85, 121413 (2012).

[39] Y. Barad, H. Eisenberg, M. Horowitz, and Y. Silberberg, Nonlinear Scanning Laser Microscopy by Third Harmonic Generation, Appl. Phys. Lett. 70, 922 (1997).

[40] N. Kumar, J. Kumar, C. Gerstenkorn, R. Wang, H.-Y. Chiu, A.L. Smirl, and H. Zhao, Third Harmonic Generation in Graphene and Few-Layer Graphite Films, Phys. Rev. B 87, 121406(R) (2013).

[41] P. Blake, E. Hill, A. Castro Neto, K. Novoselov, D. Jiang, R. Yang, T. Booth, and A. Geim, Making Graphene Visible, Appl. Phys. Lett. 91, 063124 (2007).

[42] M. A. Fanton, J.A. Robinson, C. Puls, Y. Liu, M.J. Hollander, B. E. Weiland, M. LaBella, K. Trumbull, R. Kasarda, C. Howsare, J. Stitt, and D. W. Snyder, Characterization of Graphene Films and Transistors Grown on Sapphire by Metal-Free Chemical Vapor Deposition, ACS Nano 5, 8062 (2011).

[43] Y. Wu, Y.-M. Lin, A. A. Bol, K. A. Jenkins, F. Xia, D. B. Farmer, Y. Zhu, and P. Avouris, High-Frequency, Scaled Graphene Transistors on Diamond-like Carbon, Nature (London) 472, 74 (2011).

[44] C. R. Dean, A. F. Young, I. Meric, C. Lee, L. Wang, S. Sorgenfrei, K. Watanabe, T. Taniguchi, P. Kim, K. L. Shepard, and J. Hone, Boron Nitride Substrates for High Quality Graphene Electronics, Nat. Nanotechnol. 5, 722 (2010).

[45] X. Li, Y. Zhu, W. Cai, M. Borysiak, B. Han, D. Chen, R. D. Piner, L. Colombo, and R.S. Ruoff, Transfer of Large-Area Graphene Films for High-Performance Transparent Conductive Electrodes, Nano Lett. 9, 4359 (2009).

[46] Jongho Lee, Li Tao, Yufeng Hao, Rodney S. Ruoff, and Deji Akinwande, Embedded-Gate Graphene Transistors for High-Mobility Detachable Flexible Nanoelectronics, Appl. Phys. Lett. 100, 152104 (2012).

[47] N. Petrone, I. Meric, J. Hone, and K. L. Shepard, Graphene Field-Effect Transistors with GigahertzFrequency Power Gain on Flexible Substrates, Nano Lett. 13, 121 (2013).
[48] S. Bae, H. Kim, Y. Lee, X. Xu, J.-S. Park, Y. Zheng, J. Balakrishnan, T. Lei, H. R. Kim, Y. I. Song, Y.-J. Kim, K. S. Kim, B. Özyilmaz, J.-H. Ahn, B. H. Hong, and S. Iijima, Roll-to-Roll Production of 30-Inch Graphene Films for Transparent Electrodes, Nat. Nanotechnol. 5, 574 (2010).

[49] S.-K. Lee, B. J. Kim, H. Jang, S. C. Yoon, C. Lee, B. H. Hong, J. A. Rogers, J. H. Cho, and J.-H. Ahn, Stretchable Graphene Transistors with Printed Dielectrics and Gate Electrodes, Nano Lett. 11, 4642 (2011).

[50] N. Bloembergen and P. Pershan, Light Waves at the Boundary of Nonlinear Media, Phys. Rev. 128, 606 (1962).

[51] K. Pedersen and T. G. Pedersen, Spectroscopic SecondHarmonic Generation from Silicon-on-Insulator Wafers, J. Opt. Soc. Am. B 26, 917 (2009).

[52] Robert Boyd, Nonlinear Optics (Academic, New York, 2008), 3rd ed., p. 54

[53] N. Petrone, C. R. Dean, I. Meric, A. M. van der Zande, P. Y. Huang, L. Wang, D. Muller, K. L. Shepard, and J. Hone, Chemical Vapor Deposition-Derived Graphene with Electrical Performance of Exfoliated Graphene, Nano Lett. 12, 2751 (2012).

[54] X. Li, C. W. Magnuson, A. Venugopal, R. M. Tromp, J. B. Hannon, E. M. Vogel, L. Colombo, and R. S. Ruoff, LargeArea Graphene Single Crystals Grown by Low-Pressure Chemical Vapor Deposition of Methane on Copper, J. Am. Chem. Soc. 133, 2816 (2011).

[55] A. Ferrari, J. Meyer, V. Scardaci, C. Casiraghi, M. Lazzeri, F. Mauri, S. Piscanec, D. Jiang, K. Novoselov, and S. Roth, Raman Spectrum of Graphene and Graphene Layers, Phys. Rev. Lett. 97, 187401 (2006).

[56] C. H. Lui, Z. Li, Z. Chen, P. V. Klimov, L. E. Brus, and T.F. Heinz, Imaging Stacking Order in Few-Layer Graphene, Nano Lett. 11, 164 (2011).

[57] S. Nie, W. Wu, S. Xing, Q. Yu, J. Bao, S.-S. Pei, and K. F. McCarty, Growth from below: Bilayer Graphene on Copper by Chemical Vapor Deposition, New J. Phys. 14, 093028 (2012).

[58] A.C. Ferrari, Raman Spectroscopy of Graphene and Graphite: Disorder, Electron-Phonon Coupling, Doping and Nonadiabatic Effects, Solid State Commun. 143, 47 (2007).

[59] W. Zhu, T. Low, V. Perebeinos, A. A. Bol, Y. Zhu, H. Yan, J. Tersoff, and P. Avouris, Structure and Electronic Transport in Graphene Wrinkles, Nano Lett. 12, 3431 (2012).

[60] P. B. Johnson and R. Christy, Optical Constants of the Noble Metals, Phys. Rev. B 6, 4370 (1972). 